\title{
Sex differences in dynamic spatial ability: The unsolved question of performance factors
}

\author{
María José Contreras \\ Universidad Nacional de Educación a Distancia, Madrid, Spain
}

AND

\author{
Víctor J. Rubio, Daniel Peña, Roberto Colom, and José Santacreu \\ Universidad Autónoma de Madrid, Madrid, Spain
}

\begin{abstract}
Males and females differ in several cognitive abilities, although the largest gap can be found in spatial ability. Some published studies make the claim that performance factors, which can be either subject- or task-related variables, explain these differences. However, previous studies in which performance factors were measured have focused on static spatial tests. Little is known about the role of performance factors in dynamic spatial tasks. In the present study, we analyzed a sample of 2,624 university graduates to test the role of three performance factors (response latency, response frequency, and invested time) derived from the Spatial Orientation Dynamic Test-Revised (SODT-R; Santacreu, 1999). The results showed that males and females appear to cope with the dynamic task in different ways. However, males outperformed females even when the effects of the performance factors were partialed out; that is, the assessed performance factors did not explain much of the sex-related variance. Alternative ways of measuring performance factors will be needed if they are to explain sex differences in dynamic spatial ability.
\end{abstract}

Research findings indicate that the largest average sex difference in cognitive performance derives from spatial tasks, in which males outperform females (Neisser et al., 1996). For instance, in their seminal meta-analysis, Linn and Petersen (1985) found that the male advantage in spatial performance was large for mental rotation $(d=.73)$, medium for spatial perception $(d=.44)$, and small for spatial visualization $(d=.13)$. Linn and Petersen's results came from amalgamating data obtained in both adolescents and adults. Ten years later, Voyer, Voyer, and Bryden (1995) presented a new meta-analysis enriched by new data and a more systematic incorporation of the age variable. This study showed that after the age of 18 years, the average male advantage was very similar for the three ability categories considered by Linn and Petersen. Their metaanalytically derived results revealed, for the group over 18 years of age, effect sizes of .66 for mental rotation, .48 for spatial perception, and .23 for spatial visualization.

From these research findings, the main question that arises is why average male performance is better than average female performance in spatial tasks. Halpern (2000) referred to biological and cultural explanations. However, although interesting and appealing, these explanations are largely general. For this reason, researchers have focused on more specific variables as potential causes of the average sex difference. These variables have been broadly defined as performance factors (Goldstein, Haldane, \& Mitchell, 1990) and could be described as response ten- dencies (Delgado \& Prieto, 1996) that can be measured only when the task can be approached in different ways. Performance factors could be tentatively classified in two main categories: task-related variables involved in administration procedures (e.g., instructions, time limits, scoring procedures) and subject-related variables derived from the ways people cope with these tasks (e.g., processing speed, reluctance to guess).

Goldstein et al. (1990) were the first to study performance factors, suggesting that females, as a group, could be penalized in a least two ways: by the use of scoring procedures based on the total number of correct answers instead of on correct:attempted ratios, and by measurement procedures that are applied under strong time constraints. To test their assumptions, these researchers administered the Mental Rotation Test (MRT) developed by Vandenberg and Kuse (1978) and based on Shepard and Metzler's (1971) 3 -D figures. The findings indicated that sex differences disappeared when corrected scores (correct:attempted ratios) were used as well as when no time constraints were imposed. They concluded that sex differences in spatial ability are related to measurement conditions and therefore should not be considered genuine.

Three methodological problems have been identified in Goldstein et al.'s (1990) study. First, the authors used repeated measures instead of cross-sectional designs to compare limited- and unlimited-time conditions (Voyer, 1997). Second, the researchers considered unanswered items an

M. J. Contreras, mjcontreras@psi.uned.es 
indicator of slowness and a sign of reluctance to guess (see Delgado \& Prieto, 1996). Third, further studies have had difficulty replicating the main findings of Goldstein et al. with alternative measures of spatial ability. Thus, for instance, Stumpf (1993), using a more general battery of printed spatial tests (the Spatial Test Battery; Eliot \& Stumpf, 1992), failed to replicate Goldstein et al.'s findings.

Several assumptions about how males and females cope with spatial tasks are made in the analyses of subjectrelated variables. Females are presumed to be slow and reflective, whereas males are thought to be fast and impulsive (Maccoby \& Jacklin, 1974). These differences could be attributed to several causes. The first potential cause is that females accumulate less previous experience than males with spatial tasks (Fennema \& Sherman, 1977). The second possibility is that females display poorer time management than males (Mayer, 1985). The third potential source of the sex difference is that females present low levels of confidence in their ability to solve spatial tasks (Parsons, Adler, \& Kaczala, 1982). Finally, there is the presumed unwillingness of women to guess: On average, females could be penalized by a greater reluctance to guess in conditions of uncertainty, as is indicated by their higher levels of omissions (Parsons et al., 1982).

Some researchers have found evidence suggesting differences in guessing behavior between females and males in spatial tasks (Goldstein et al., 1990; Stumpf, 1993). However, these findings are not supported when rotation tests other than the MRT are used or when methodological control is increased (Voyer, Rodgers, \& McCormick, 2004). Thus, for instance, Delgado and Prieto (1996) administered two different mental rotation tasks: the Rotation of Solid Figures (RFM) Test (Yela, 1968) and the reduced version of the spatial relations test from the Differential Aptitude Test battery (DAT-SR; Bennett, Seashore, \& Wesman, 1974; Técnicos Especialistas Asociados, 1990). They also distinguished unanswered but attempted items (omissions) from unanswered and not attempted items. Half of their participants received instructions emphasizing speed, whereas the other half were assigned to a condition in which accuracy was encouraged. Their findings indicated that there were no sex differences in the number of omissions (e.g., no differences in guessing behavior). Furthermore, the females made more errors than the males with both raw and ratio scoring procedures, a result that was found in both accuracy and speed conditions. These results substantially agree with Voyer and colleagues (Voyer, 1997; Voyer et al., 1995), who did not find sex differences in guessing behavior, although they did find processing speed, response latency, and training level to be relevant.

In the studies considered above, static spatial tests were analyzed. However, since the publication of the seminal study by Hunt, Pellegrino, Frick, Farr, and Alderton (1988), researchers have clearly distinguished between static and dynamic spatial tests.

Traditional ability tests measure what an examinee already knows or what he or she actually does. In contrast, dynamic testing emphasizes what the examinee would be able to do or how he or she could do it (Embretson, 2000). Ability measured with dynamic tests depends on the ex- aminee's previous responsiveness to the assessment conditions (Grigorenko \& Sternberg, 1998). In other words, a dynamic item represents a setting that changes according to a time function, as does the response. Dynamic tests place emphasis on the psychological process involved in the task and not just on performance (Grigorenko \& Sternberg, 1998). Regarding spatial ability, dynamic tasks are used to test the ability to perceive and extrapolate real motion, to predict trajectories of moving objects, and to estimate arrival times of two or more objects (Colom, Contreras, Botella, \& Santacreu, 2002; Contreras, Colom, Hernández, \& Santacreu, 2003; D’Oliveira, 2004; Law, Pellegrino, Mitchell, et al., 1993; Pellegrino \& Hunt, 1989; Pellegrino, Hunt, Abate, \& Farr, 1987).

Performance factors may also be at play in dynamic computerized tasks, such as the Spatial Orientation Dynamic Test-Revised (SODT-R; Santacreu, 1999), which can be approached in different ways. However, to the best of our knowledge, there are no published studies considering the role of performance factors on these dynamic tasks. Published evidence supports the SODT-R as a good way of measuring dynamic spatial ability. Colom et al. (2002) found that measures of visualization, spatial relations (SR), and dynamic spatial performance (DSP) show high loadings on a powerful hierarchical higher order factor designated as $G v$ (general spatial ability). Colom et al. (2002) showed that (1) there is a single strong source of variance and (2) it is difficult to separate specific spatial tests by construct. Colom, Contreras, Shih, and Santacreu (2003) considered a broad sample of tests measuring reasoning, visualization, SR, and DSP. The SODT-R, tapping central spatial mental processes, emerged as a very good measure of Gv.

It is worth emphasizing that this task comprises several different potential performance factors that cannot easily be compared with those considered for static tasks - namely, time pressure and guessing. Rather, performance factors that could be taken into account in an analysis of the results of dynamic spatial tests such as this one are related to the three main components of the task: planning, orientation, and visual-motor coordination. In the present work, three subject-related performance factors were considered: response latency (RL), response frequency (RF), and invested time (IT). Each one is related to one of the main components of the task.

More specifically, RL concerns the planning action a person carries out when confronting the task. It is also related to the impulsivity an individual shows, according to the definition and assessment of the reflection-impulsivity construct that several authors have provided (Kagan, Rosman, Day, Albert, \& Phillips, 1964; Salkind \& Wright, 1977). RF informs about efficiency in solving the task (independently of effectiveness) and is closely related to the orientation component of a spatial task. This efficiency has been emphasized as an aspect in high-complexity jobs (Ackerman \& Kanfer, 1993). Finally, IT is related to the visual-motor coordination an individual shows in performing the task and is remotely associated to double-checking, used as a performance factor in previous studies.

Given that several studies have demonstrated that males, on average, have higher levels of DSP than females 
(Contreras, Colom, Shih, Alava, \& Santacreu, 2001; Law, Pellegrino, \& Hunt, 1993; Saccuzzo, Craig, Johnson, \& Larson, 1996; Schiff \& Oldak, 1990), here we explore two related questions: (1) Are there sex differences in some basic DSP factors? and, if so, (2) can these differences explain the documented sex difference (Contreras et al., 2001) in overall performance on this dynamic spatial task?

We adopted as a working hypothesis the idea that performance factors ("doing the task in different ways") may also explain the sex difference. Although the task-related variables involved in administration procedures might play a role in explaining the resulting sex difference, here we limit our exploration to the role of three subject-related factors derived from the ways people cope with the SODT-R task.

\section{METHOD}

\section{Participants}

The sample comprised 2,624 applicants for an ab initio air traffic control (ATC) training course. As required by the selection process, all of them were university graduates: Nineteen percent were graduates in the humanities, $37 \%$ in the social sciences, $24 \%$ in the natural sciences, and $20 \%$ in engineering. Furthermore, they were all applying for the first time. There were 1,291 females (49\%) and 1,333 males $(51 \%)$. The sample was randomly selected from the total population of applicants, ensuring that the numbers of males and of females within each educational group were very similar. The mean age of the females was 27.79 years $(S D=3.86)$, whereas the mean age of the males was 28.22 years $(S D=4.10)$.

\section{Measures and Procedure}

The participants completed the SODT-R - a computer-based spatial dynamic test - along with other tests administered to predict their performance in the ATC training course for which they were applying.

The SODT-R assesses the ability to guide two moving dots toward a destination. The course of each dot adopts a value of $0^{\circ}-359^{\circ}$ on the plane of the screen, with the top of the screen being at $0^{\circ}$.

Figure 1 presents an example of a trial in which two dots, each of a different color, have previously appeared in different positions and are advancing along their initial courses. Both dots initially appear at the same distance from the target, have an original course that forms a $90^{\circ}$ angle with regard to the correct course, and advance at a constant speed. On the screen, one can see the dots and the tracks they make. The track consists of a gray dot that shows where the colored dot was at the previous moment. The track helps the participant to estimate the current course of the colored dot.

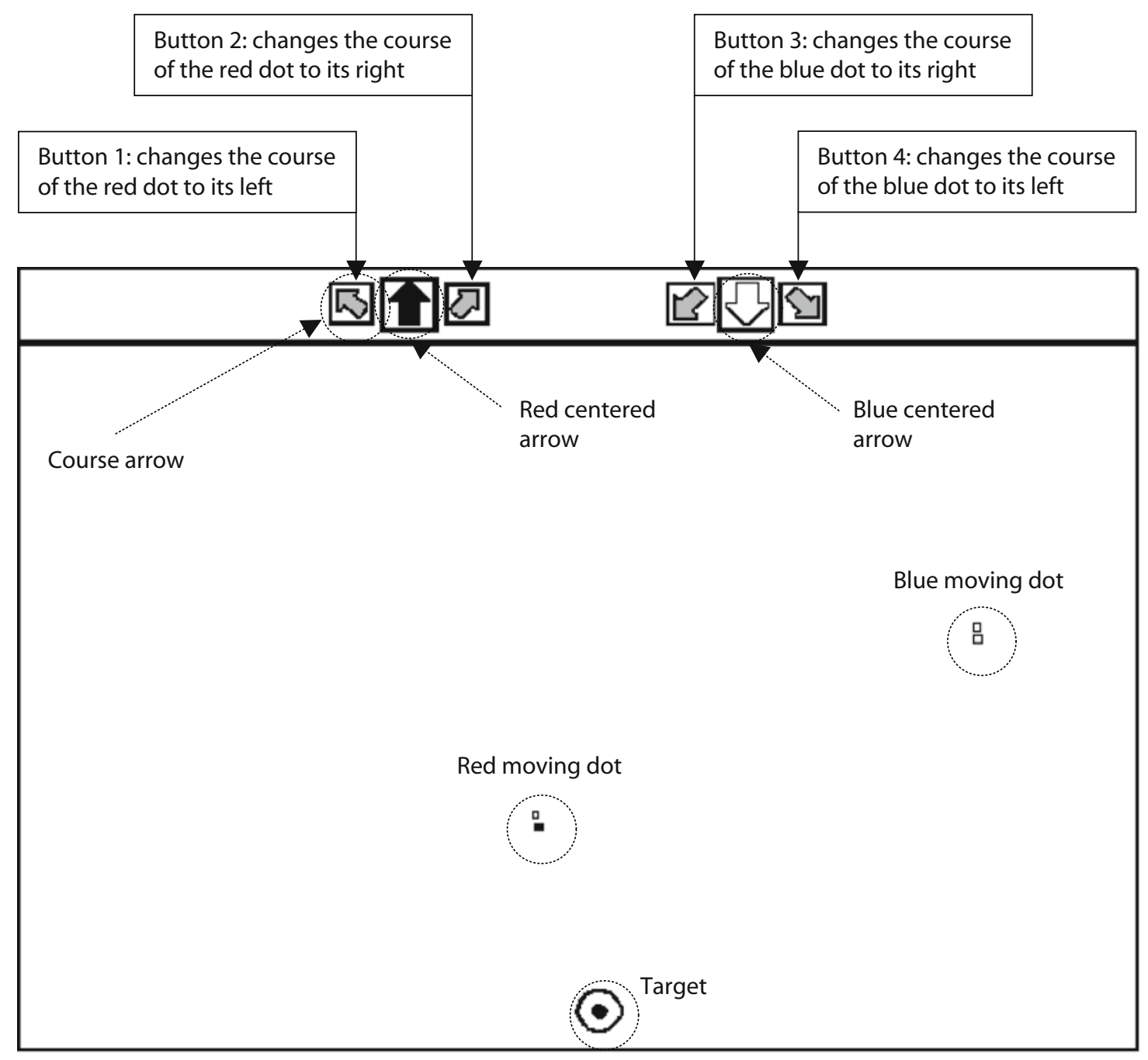

Figure 1. Example of a trial from the SODT-R. 
A translation of the written instructions given to the participants follows (see Figure 1 to better understand what the instructions are describing):

In this task, two different colored points or dots moving on an established course will appear on your computer screen. There will be two boxes with arrows in the upper area of the screen. These arrows will allow you to modify the course of the dots. An arrow that is the same color as the associated dot appears in a centered box. Two more arrows also appear beside it. By pressing repeatedly on them, you will be able to modify the course of the dot that is the same color as the centered arrow.

Your goal will be to guide the dots toward the target in a minimum amount of time. You can adjust the course of the dots by pressing repeatedly on the arrows beside the centered arrow of the same color. The color of the centered arrow corresponds to the associated dot, and the arrows beside it indicate the course that the dot will take if you press on them.

The program sets an original position and a course for the dot that can be modified by pressing the arrow buttons. If the course is not modified, the dot advances across the screen in accordance with the course specified in the configuration and can even go off the screen. Participants can make each of the dots (signaled by the arrow centered between the two course arrow buttons) turn to the right or left by clicking with the mouse on the respective course arrow button. The course arrow buttons and the positions of the centered arrows at the top of the screen do not change from trial to trial. The direction (up or down) of the centered arrow is important because it determines the direction that the dot will move when the adjacent arrow buttons are pushed. That is, as is shown in Figure 1, the button to the right of the "up" arrow moves the red dot to its right, and the button to the left of the "down" arrow moves the blue dot to its right. Repeatedly pressing one of the course arrow buttons changes the course of the dot (to the left or to the right, depending on the direction of the centered arrow) by $10^{\circ}$ per click. Every time an arrow is pressed, it changes the direction of the dot, which continues following its new course until the button is pressed again. For each of the trials, participants can see (1) the two dots advancing in accordance with their current course, (2) how each one has been modified (through pressing of the respective course arrow buttons), and, therefore, (3) their courses with regard to the target, which is also always visible.

The test comprises a total of 13 trials. The first four trials are practice trials and are used so that participants can train in handling and understanding the set of tasks they need to perform. These first four trials are not considered when calculating the participant's score. Trial 1 lasts for $50 \mathrm{sec}$, and this time period enables the participant to know whether or not the moving dots arrive at the requested destination because it is approximately the time it takes for the dots to reach the target. However, the remaining trials last $20 \mathrm{sec}$ each, so participants do not have enough time to see the dot arrive. In three experimental trials, the dots begin to move from the top of the computer screen, whereas the destination is on the bottom (Figure 1 shows an example of this type of trial). In another three experimental trials, the dots begin to move from the left of the computer screen, whereas the destination is on the right. Finally, in the remaining three experimental trials the dots begin to move from the right of the computer screen, whereas the destination is on the left. The three types of trials are mixed in a programmed sequence that is always the same (destination at bottom, left, bottom, right, bottom, left, right, left, right, bottom, right, bottom, and left; the experimental trials are the last nine in the sequence). The participant's first task is to decide which course arrow button to press first to change the course of one of the dots, which move at the same speed. The speed of the moving dots is constant. If their course is not corrected by pressing the course arrow button repeatedly, the dots follow their original course, moving away from the target.

Obviously, some participants may get around this calculation and press any of the course arrow buttons at random and act according to the changes in the course of one of the two moving dots. In this way, the course of the dot may be modified as desired, in which case it will have to be adjusted only until the dot is on the correct course. If, on the other hand, the dot modifies its course in the direction opposite to the desired one, the participant can modify the course of the moving dot just by pressing on the other course arrow button. Participants who concentrate on the effect of each press of the course arrow buttons may press them more often than participants who work out beforehand which button to press. If a participant calculates beforehand which course arrow button to press, he or she will generally spend less time pressing the buttons because fewer presses will be needed to put each dot on the correct course. There are also participants who spend a large amount of time adjusting the course of the dot, constantly reviewing its route with regard to the position of the target. Therefore, the number of course corrections is independent of the time spent pressing the course arrow buttons.

For the task of changing the direction of the dots so that they can be guided to their target, participants are given a limited but sufficient time. It is important for participants to distribute their time and correct the courses of the two dots; otherwise, at the end of the trial one of the dots might be correctly oriented toward the target whereas the other maintains its original route and may even go off the screen.

At all times, the program registers the positions of the dots on the screen and their courses at each moment. The position is determined by the values of the $x$-, $y$-coordinates on the screen, and the course is a value ranging from $0^{\circ}$ (at the top of the screen) to $359^{\circ}$, as shown on a compass. The program, using the positions of the dots at the end of the trial, computes what course should have been taken to reach the target (i.e., the correct course). It then calculates the average course of the two dots at the moment when the trial finishes and compares the average course with the correct course. This difference, expressed in degrees of absolute value, is the deviation score that we use in our data analysis. For example, in Figure 1 the blue dot, which is moving on a $180^{\circ}$ course, will end the trial with a deviation score of $46^{\circ}$ with respect to the correct course $\left(226^{\circ}\right)$. The degrees of deviation for both dots on each trial are averaged to get the deviation score. Therefore, low performance implies large average deviations, whereas high performance implies small average deviations.

The program also calculates three task-related variables that were taken as performance factors potentially influencing spatial performance:

Response latency (RL) is the time taken until the participant presses any of the course arrow buttons for the first time in each trial. Presumably, this time is devoted to figuring out the initial orientation of the incoming dots, predicting the degree of adjustment needed, or both. It can be predicted that overall performance will be associated with large RLs.

Response frequency $(\mathrm{RF})$ is the number of times the course arrow buttons are pressed in each trial. Because larger numbers of buttonpresses are associated with trial-and-error behavior leading to repeated over- and undercorrection of the course, it can be predicted that overall performance will be associated with smaller RFs.

Invested time (IT) is the time from the first press (e.g., at $5 \mathrm{sec}$ ) to the last one (e.g., at $15 \mathrm{sec}$ ) in each trial. It is assumed that participants stop buttonpressing when they are satisfied with the course. It can be predicted that overall performance will be associated with smaller ITs.

Table 1

Descriptive Statistics for Males and Females and Standardized Sex Differences (Cohen's $d$ )

\begin{tabular}{lrrrrrr}
\hline & \multicolumn{2}{c}{ Females } & & \multicolumn{2}{c}{ Males } & \\
\cline { 2 - 3 } \multicolumn{1}{c}{ Measure } & \multicolumn{1}{c}{$M$} & \multicolumn{1}{c}{$S D$} & & \multicolumn{1}{c}{$M$} & \multicolumn{1}{c}{$S D$} & \\
\hline Deviation (degrees) & 53.16 & 34.00 & & 27.01 & 24.75 & .81 \\
Response latency (sec) & 1.92 & 0.93 & & 2.30 & 0.73 & .51 \\
Response frequency & 40.31 & 13.82 & & 33.85 & 10.49 & .56 \\
Invested time (sec) & 18.24 & 1.34 & & 17.44 & 1.39 & .56 \\
\hline
\end{tabular}




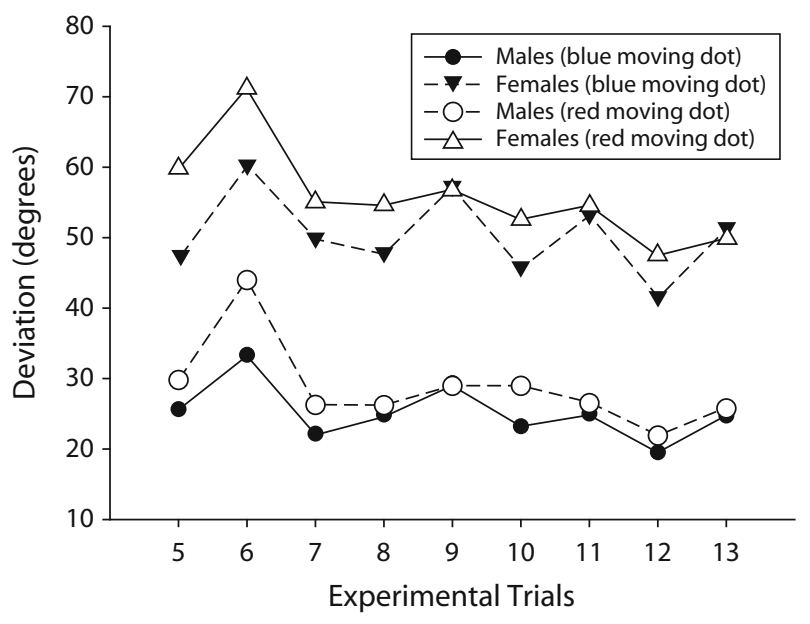

Figure 2. Trial-by-trial analysis of performance for males and females on the red and blue dots.

We should remark that the participants were instructed to minimize the deviation of the incoming moving dots, with no mention of RL, RF, or IT.

\section{RESULTS}

Table 1 shows the descriptive statistics for the males and the females as well as the standardized sex difference (d). The reliability of the SODT-R was .91 (Cronbach's alpha).

ANOVAs ${ }^{1}$ showed that there are significant differences $(p<.01)$ between the sexes for deviation $\left[F_{\mathrm{BF}}(1,2387.61)=\right.$ $\left.504.3 ; \eta^{2}=.16\right]$, RL $\left[F_{\mathrm{BF}}(1,2425.8)=128.95 ; \eta^{2}=\right.$ $.07]$, RF $\left[F_{\mathrm{BF}}(1,2407.12)=181.32 ; \eta^{2}=.05\right]$, and IT $\left[F_{\mathrm{BF}}(1,2622)=221.65 ; \eta^{2}=.08\right]$.

The males displayed smaller deviation indices than the females. Furthermore, their RLs were larger, whereas their RFs and ITs were both smaller. Thus, the males, on average, took more time to appraise the first orientation of the incoming moving dots (higher RL) and then invested less time (smaller IT) and made fewer arrow clicks (smaller $\mathrm{RF}$ ) to complete a given trial.

We carried out a trial-by-trial analysis to determine whether the sex difference was present from the beginning or emerged at some point in the series of trials. Furthermore, we analyzed the data for the two moving dots separately. The mean deviation score could mask the fact that accuracy might have been much higher for one (better attended) dot than for the other. Perhaps a fair number of participants did not have time to complete their adjustments for one of the dots, and this might possibly have occurred more often among the females than among the males if females are slower or more reflective.

Figure 2 shows performance separately for males and females for both red and blue dots. Independent sample $t$ tests showed that the males outperformed the females across every trial for each type of $\operatorname{dot}($ all $p \mathrm{~s}<.01)$. There is no indication that performance was better for one dot than for the other or that the sex difference was limited to any subset of trials. Basically, the patterns of performance for the males and the females were similar. Only the absolute levels of performance differed.

Table 2 shows the correlations among deviation, RL, $\mathrm{RF}$, and IT, separately for the males and the females.

For both the males and the females, deviation correlated negatively with RL (the smaller the deviation, the greater the RL) but positively with IT and RF (the larger the deviation, the greater the RF and IT). Furthermore, RL correlated negatively with IT and RF. Therefore, performance factors are associated with overall DSP in the predicted direction. Table 2 also shows the differences in correlations measured in the males and the females through Fisher's (1915) $z$ test. There were significant differences $(p<.01)$ for all pairs of correlations except for the deviation-RF pair. The result indicates that relations between performance factors and deviation are not the same for men as they are for women.

An ANCOVA was conducted to further explore the relationship between performance factors (RL, RF, and IT) and overall spatial performance (deviation). The males still outperformed the females in overall spatial performance after we partialed out the effect of performance factors $\left[F(1,2613)=285.05 ; \eta^{2}=.098\right]$.

\section{DISCUSSION}

Several studies have focused on performance factors to account for gender differences in static spatial tasks (Delgado \& Prieto, 1996; Goldstein et al., 1990; Stumpf, 1993; Voyer, 1997; Voyer et al., 2004; Voyer \& Saunders, 2004). Nevertheless, as Voyer (1997) stated,

Researchers using the MRT in future studies are likely to find this approach useful in refining their interpretations. It is, however, important to remember that the present results only apply to the MRT and cannot be generalized to other tasks. Such generalizations would require an adaptation of the present procedure to other spatial tests. (p. 274, italics added)

The statement above provided the main motivation for the present study: We wanted to explore the potential role of several performance factors in the execution of a dynamic spatial test. Although several published reports have considered the role of task- and subject-related variables in an attempt to explain the large average sex difference in

Table 2

Correlations for Males and Females and Differences for the Pairs of Dependent Variables Using Fisher's $z$ Transformation

\begin{tabular}{lccc}
\hline Dependent Variables & Females & Males & Sex Difference $(z)$ \\
\hline Deviation-RL & -.307 & -.199 & 2.96 \\
Deviation-RF & .257 & .302 & -1.25 \\
Deviation-IT & .411 & .288 & 3.59 \\
RL-RF & -.732 & -.620 & 5.32 \\
RL-IT & -.544 & -.301 & 7.65 \\
RF-IT & .565 & .456 & 3.78 \\
\hline
\end{tabular}

Note-RL, response latency; RF, response frequency; IT, invested time. All correlations except for that of the deviation-RF pair are significant $(p<.01)$. 
static spatial ability tasks, this is the first report to include an analysis of the role of several performance factors in a dynamic spatial task. For this very reason, the findings could be considered as derived from a new research development (Contreras et al., 2003; D'Oliveira, 2004). The present study focuses on specific subject-related variables related to how individuals cope with a particular dynamic spatial task. Although the instructions were the same for all the participants, different participants adopted different strategies. Some of them changed the directions of the moving dots only when they were certain of the correct movement. Other participants displayed a kind of impulsive behavior, changing the directions of the moving dots from the very beginning of the trial. They followed what can be called a "trial-and-error" strategy, clicking on any arrow to see if the dot moves in the correct direction and, if it did not, then clicking on the other arrow. These different styles of approach could be related to spatial performance, which would help to account for the observed overall sex differences.

The novelty of the present task makes it difficult to compare the results observed here with those of previous studies. Some performance factors previously studied (e.g., double-checking the answers or reluctance to guess) are not directly applicable to dynamic spatial tasks. For instance, it should be noted that a reluctance to guess cannot be assessed in such tasks because it is not possible to leave items blank. However, we can analyze the impact of some other performance factors related to RLs, time pressure, or response styles (e.g., impulsiveness, slowness) that could appear similar in both static and dynamic tasks.

After an in-depth analysis of the components of the SODT-R task, we selected three performance factors: (1) RL, which was thought to reflect the time devoted to tracking the initial orientation of the incoming dots; (2) RF, which was used to assess the number of times the arrows were clicked in attempts to guide the moving dots to a particular destination; and (3) IT, which was measured in terms of the time spent on efficiently guiding the moving dots, from the first arrow click to the last arrow click. Each of the three performance factors was related to one of the major components of the task: planning, orientation, and visual-motor coordination, respectively.

The main reasons underlying the selection of these performance factors were stated in the form of specific predictions, taken as a starting point the integrated model of reflection-impulsivity and cognitive efficiency proposed by Salkind and Wright (1977). The authors proposed in their model that both latency and error scores are products of the interaction between efficient information processing and the task-specific choices. Applying the efficiency model to our specific task, we predicted that deviation would be associated with larger RLs, smaller RFs, and shorter ITs. Participants showing low deviation scores would also show small RFs and short ITs but high RLs. Regarding the average difference between males and females, it was predicted that the difference would disappear if RLs, RFs, and ITs were in fact responsible for the sex difference.

The results showed that males and females appear to cope with the dynamic task in different ways. Males dis- play larger RLs, smaller RFs, and shorter ITs than females. Thus, it can be presumed that, on average, males take more time to mentally follow the moving dots before taking any action to effectively direct them to their destination. Moreover, once they have a dynamic picture of the moving dots, they invest less time and use fewer clicks than females to effectively guide the moving dots to their destination. Differences in patterns of correlations obtained in both sexes reinforce the thesis that males and females cope with the SODT-R task in different ways. However, trial-by-trial analysis showed that females had the same pattern of spatial performance as males. Although accuracy was much better for males, both sexes completed their adjustments for the two dots. There was no evidence to support the hypothesis that accuracy might have been much better for one (better attended) dot than for the other.

Voyer et al. (1995) suggested that procedural differences are an important source of variance in the magnitude of sex differences. Can the overall difference in the dynamic spatial task be attributed to these performance factors? According to our findings, the answer is negative. Males still have an advantage when these performance factors are partialed out. Therefore, although there are sex differences on the analyzed performance factors, these differences do not appear to be the underlying cause of the observed overall sex difference in DSP. Obviously, RL, RF, and IT are not the only possible performance factors that can be assessed in the SODT-R. We must acknowledge that there may be other performance factors involved in the task. We are currently redesigning the computer program for further studies in order to look into some other performance factors, such as how often the task changes (guiding one dot or the other), because we have noticed that some participants frequently switch from one dot to the other, whereas others take care of one of them first and then move on to the other.

In conclusion, the findings reported here support the statement that the performance factors considered do not account for sex differences in dynamic spatial ability. Stumpf (1993) made the following conclusion:
Although it is clear that performance factors cannot explain much of the gender-related variance, this does not mean that they should be ignored in further stud- ies. On the contrary, a more precise definition and a more detailed knowledge of performance factors and alternative ways of measuring them are worthy of continued research. Future studies on performance factors should focus more closely on the process of solving spatial tasks. Presenting items individually and assessing the latencies of subjects' responses, as well as using ratings to assess subjects' levels of con- fidence when they take spatial tests are promising ways of operationally defining performance factors more precisely. (pp. 833-834)

This could likewise be the main conclusion of the present paper. We are convinced that analysis of performance factors is an appropriate approach for studying the process of solving spatial tasks. Future studies may be able to resolve the question of the specific role of performance factors 
in the explanation of sex differences in dynamic spatial ability.

\section{AUTHOR NOTE}

This research was partially supported by the AENA-UAM/785001 project. The authors thank Veronica J. Dark for her stimulating, patient, and conscientious comments and suggestions as action editor of this article, which have very much enriched this work. Correspondence concerning this article should be addressed to M. J. Contreras, Facultad de Psicología, Universidad Nacional de Educación a Distancia, Juan del Rosal, 10, Madrid, Spain 28040 (e-mail: mjcontreras@psi.uned.es).

Note-This article was accepted by the previous editorial team, when Colin M. MacLeod was Editor.

\section{REFERENCES}

Ackerman, P. L., \& Kanfer, R. (1993). Integrating laboratory and field study for improving selection: Development of a battery for predicting air traffic controller success. Journal of Applied Psychology, 78, 413-432.

Bennett, G. K., Seashore, H. G., \& Wesman, A. G. (1974). Differential Aptitude Test manual (5th ed.). New York: Psychological Corp.

Colom, R., Contreras, M. J., Botella, J., \& Santacreu, J. (2002). Vehicles of spatial ability. Personality \& Individual Differences, 32 903-912.

Colom, R., Contreras, M. J., Shih, P. C., \& Santacreu, J. (2003). The assessment of spatial ability with a single computerized test. European Journal of Psychological Assessment, 19, 92-100.

Contreras, M. J., Colom, R., Hernández, J. M., \& Santacreu, J. (2003). Is static spatial performance distinguishable from dynamic spatial performance? A latent-variable analysis. Journal of General Psychology, 130, 277-288.

Contreras, M. J., Colom, R., Shih, P. C., Alava, M. J., \& Santacreu, J. (2001). Dynamic spatial performance: Sex and educational differences. Personality \& Individual Differences, 30, 117-126.

Delgado, A. R., \& Prieto, G. (1996). Sex differences in visuospatial ability: Do performance factors play such an important role? Memory \& Cognition, 24, 504-510.

D'Oliveira, T. C. (2004). Dynamic spatial ability: An exploratory analysis and a confirmatory study. International Journal of Aviation Psychology, 14, 19-38.

Eliot, J., \& StumpF, H. (1992). CTY spatial test battery. Baltimore: Center for Talented Youth.

Embretson, S. E. (2000). Multidimensional measurement from dynamic tests: Abstract reasoning under stress. Multivariate Behavioral Research, 35, 505-542.

Fennema, E., \& Sherman, J. (1977). Sex-related differences in mathematics achievement, spatial visualization and affective factors. American Educational Research Journal, 14, 51-71.

FISHER, R. A. (1915). Frequency distribution of the values of the correlation coefficient in samples from an indefinitely large population. Biometrika, 10, 507-521.

Goldstein, D., Haldane, D., \& Mitchell, C. (1990). Sex differences in visual-spatial ability: The role of performance factors. Memory \& Cognition, 18, 546-550.

Grigorenko, E. L., \& Sternberg, R. J. (1998). Dynamic testing. Psychological Bulletin, 124, 75-111.

Halpern, D. F. (2000). Sex differences in cognitive abilities (3rd ed.) Mahwah, NJ: Erlbaum.

Hunt, E., Pellegrino, J. W., Frick, R. W.,Farr, S. A., \&Alderton, D. (1988). The ability to reason about movement in the visual field. Intelligence, 12, 77-100.

Kagan, J., Rosman, B. L., Day, D., Albert, J., \& Phillips, W. (1964). Information processing in the child: Significance of analytic and reflective attitudes. Psychological Monographs: General \& Applied, 78 (1, Whole No. 578)

LaW, D. J., Pellegrino, J. W., \& Hunt, E. B. (1993). Comparing the tortoise and the hare: Gender differences and experience in dynamic spatial reasoning tasks. Psychological Science, 4, 35-40.
Law, D. J., Pellegrino, J. W., Mitchell, S. R., Fischer, S. C., McDonald, T. P., \& Hunt, E. B. (1993). Perceptual and cognitive factors governing performance in comparative arrival-time judgments. Journal of Experimental Psychology: Human Perception \& Performance, 19, 1183-1199.

Linn, M. C., \& Petersen, A. C. (1985). Emergence and characterization of sex differences in spatial ability: A meta-analysis. Child Development, 56, 1479-1498.

MACCOBY, E. E., \& JACKLIN, C. N. (1974). The psychology of sex differences. Stanford: Stanford University Press.

MAYER, R. (1985). Mathematical ability. In R. J. Sternberg (Ed.), Human abilities: An information-processing approach (pp. 127-150). New York: Freeman.

Neisser, U., Boodoo, G., Bouchard, T. J., JR., Boykin, A. W., Brody, N., CECI, S. J., ET AL. (1996). Intelligence: Knowns and unknowns. American Psychologist, 51, 77-101.

Parsons, J. E., Adler, T. F., \& Kaczala, C. M. (1982). Socialization of achievement attitudes and beliefs: Parental influences. Child Development, 53, 310-321.

Pellegrino, J. W., \& Hunt, E. B. (1989). Computer-controlled assessment of static and dynamic spatial reasoning. In R. F. Dillon \& J. W. Pellegrino (Eds.), Testing: Theoretical and applied perspectives (pp. 174-198). New York: Praeger.

Pellegrino, J. W., Hunt, E. B., Abate, R., \& Farr, S. (1987). A computer-based test battery for the assessment of static and dynamic spatial reasoning abilities. Behavior Research Methods, Instruments, \& Computers, 19, 231-236.

Saccuzzo, D. P., Craig, A. S., Johnson, N. E., \& Larson, G. E. (1996). Gender differences in dynamic spatial abilities. Personality \& Individual Differences, 21, 599-607.

SALKIND, N. J., \& WRIGHT, J. C. (1977). The development of reflectionimpulsivity and cognitive efficiency: An integrated model. Human Development, 20, 377-387.

SANTACREU, J. (1999). SODT-R and SVDT-R: Dynamic computerized test for the assessment of spatial ability (revised versions) (Technical Report). Madrid: Autonomous University of Madrid.

SCHIFF, W., \& OldAK, R. (1990). Accuracy of judging time to arrival: Effects of modality, trajectory and gender. Journal of Experimental Psychology: Human Perception \& Performance, 16, 303-316.

ShePard, R. N., \& Metzler, J. (1971). Mental rotation of threedimensional objects. Science, 171, 701-703.

StUmPF, H. (1993). Performance factors and gender-related differences in spatial ability: Another assessment. Memory \& Cognition, 21, 828-836.

TÉCnicos Especialistas Asociados (1990). DAT. Tests de aptitudes diferenciales: Manual [DAT. Differential aptitudes tests: Manual] (10th ed.). Madrid: TEA.

VAndenberg, S. G., \& Kuse, A. R. (1978). Mental rotations, a group test of three-dimensional spatial visualization. Perceptual \& Motor Skills, 47, 599-604.

VOYER, D. (1997). Scoring procedure, performance factors, and magnitude of sex differences in spatial performance. American Journal of Psychology, 110, 259-276.

Voyer, D., Rodgers, M. A., \& McCormick, P. A. (2004). Timing conditions and the magnitude of gender differences on the Mental Rotations Test. Memory \& Cognition, 32, 72-82.

Voyer, D., \& Saunders, K. A. (2004). Gender differences on the mental rotations test: A factor analysis. Acta Psychologica, 117, 79-94.

Voyer, D., Voyer, S., \& Bryden, M. P. (1995). Magnitude of sex differences in spatial abilities: A meta-analysis and consideration of critical variables. Psychological Bulletin, 117, 250-270.

Yela, M. (1968). Rotación de figuras macizas: Manual (Rotation of solid figures: Manual). Madrid: TEA.

\section{NOTE}

1. The Brown-Forsythe (BF) test for homogeneity of variance has been used.

(Manuscript received March 30, 2004; revision accepted for publication January 31,2006 .) 